\title{
Optical Waveguide Beam Splitters Based on Hybrid
}

\section{Metal-dielectric-semiconductor Nanostructures}

Yunyun Li", Junwu Liang, Qinglin Zhang ${ }^{\mathrm{a}, *}$, Zidong Zhou ${ }^{\mathrm{a}}$, Honglai Li ${ }^{\mathrm{a}}$, Xiaopeng

Fan $^{a}$, Xiaoxia Wanga, Peng Fan ${ }^{a}$, Yankun Yang ${ }^{a}$, Pengfei Guo ${ }^{a}$, Xiujuan Zhuanga ${ }^{a}$ Xiaoli

Zhu ${ }^{a}$, Lei Liaoc, Anlian Pan ${ }^{\text {a,* }}$

${ }^{a}$ Key Laboratory for Micro-Nano Physics and Technology of Hunan Province, College of Physics and Microelectronics Science, Hunan University, Changsha 410082, China

${ }^{b}$ School of Physical Science and Technology Engineering, Guangxi Universities Key Lab of Complex System Optimization and Big Data Processing, Yulin Normal Universty, Yulin, 537400, China

${ }^{c}$ Department of Physics and Key Laboratory of Artificial Micro- and Nano-structures of Ministry of Education, Wuhan University, Wuhan 430072, P. R. China

* Corresponding author. Tel.: 0086-731-88820932; fax: 0086-731-88822332.

E-mail addresses: qinglin.zhang@hnu.edu.cn (Q.Zhang), anlian.pan@hnu.edu.cn (A.Pan).

Abstract: Miniature integration is desirable for the future photonics circuit. Low-dimensional semiconductor and metal nanostructures is the potential building blocks in compact photonic circuits for their unique electronic and optical properties. In this work, a hybrid metal-dielectric-semiconductor nanostructure is designed and fabricated to realizing a nano-scale optical waveguide beam splitter, which is constructed with the sandwiched structure of a single $\mathrm{CdS}$ nanoribbon/ $/ \mathrm{HfO}_{2}$ thin film/Au nanodisk arrays. Micro-optical investigations reveal that the guided light outputting at the terminal end of the CdS ribbon is well separated into several light 
spots. Numerical simulations further demonstrate that the beam splitting mechanism is attributed to the strong electromagnetic coupling between the Au nanodisks and light guided in the nanoribbon. The number of the split beams (light spots) at the terminal end of the nanoribbon is mainly determined by the number of the $\mathrm{Au}$ nanodisk rows, as well as the distance of the blank region between the nanodisks array and the end of the CdS ribbon, owing to the interference between the split beams. These optical beam splitters may find potential applications in high-density integrated photonic circuits and systems.

Keywords: metal-dielectric-semiconductor; optical waveguide beam splitter; surface plasmons; interference; light spot; numerical simulation

\section{Introduction}

Recently, Miniaturization of optical components or devices is necessary in developing integrated photonic circuit, which has led to the great advances in developing various compact optical elements, such as nanolasers [1-4], optical switching [5] and filter [6]. Beam splitter in the nano-micro scale is one of the key elements for photonic circuits, and is commonly used in many kinds of optical systems for optical information processing, optical computing, holography, and metrology [7-15]. Surface plasmons (SPs), a coherent oscillation of the surface conduction electrons excited by electromagnetic radiation, has series of special optical properties, including sub-wavelength confinement of light, electromagnetic enhancement and polarization sensitivity [16-24]. For these particular properties of SPs, low dimensional noble metal nanostructures, for example, nanoparticles, 
nanowires and nanofilms, have enabled a vast array of applications, including surface-enhanced spectroscopies, biological/chemical sensing, and optical information processing [25-29]. However, optical information transferred by SPs is not an ideal candidate owing to the large intrinsic ohmic damping of the metal [30]. Fortunately, one-dimensional (1D) semiconductor nanostructures, are not only promising building blocks in assembling compact optical elements, but also the bridging of the nanoscopic and macroscopic world, for their unique optical properties and high-aspect-ratio [31-36]. Comparing with other dimensional nanostructures such as quantum dot and thin film, 1D semiconductor nanostructure (nanowires/nanoribbons) is easier to be manipulated and can offer a more excellent platform for tight confining and guiding light at sub-wavelength scale in one dimension, because they can confine photons in two dimensions and let the light propagate freely in the third dimension [37-40]. CdS nanoribbon is an optimal waveguide cavity for its rectangular cross section with a well-defined geometry, relatively high refractive index and flat surface [41]. Additionally, due to the high luminous efficiency, the light emitted from the CdS nanoribbon can be directly guided in the ribbon, which makes the waveguide avoid the light coupling. Utilizing the strong electromagnetic coupling between the optical waveguide in the 1D semiconductor nanostructures and low dimensional noble metal nanostructures to induce SPs can give rise to abundant optical properties and even create a new path to invent better-performance optical elements [42-45]. Here, we designed and fabricated a novel sandwich-like hybrid nanostructure for the first time, which composed by $\mathrm{Au}$ nanodisks array covering on a CdS nanoribbon, with a thin 
$\mathrm{HfO}_{2}$ film as the intermediate dielectric layer. Due to the electromagnetic coupling of SPs and optical waveguide in the nanoribbon, the guided light in the ribbon can be isolated and separated by the Au nanodisks array rows. Meanwhile, the increase of blank region distance between the nanodisks array and the terminal end of the CdS ribbon results in the multi-beam interference in the nanoribbon. The number of the split beams (light spots) is determined by the row number of the Au nanodisk array and the distance of the blank region between the Au nanodisk arrays and the ribbon end. For construction of a novel optical waveguide beam splitter at nano/micro-scale, these results may take a significant step toward the goal and find a new way to realize small footprint photonic circuits.

\section{Fabrication}

In this study, CdS nanoribbons with length of several tens micron and width of several micron were synthesized through a chemical vapor deposition (CVD) route as previously reported in the literature [46]. $\mathrm{Si} / \mathrm{SiO}_{2}$ (thickness of $\mathrm{SiO}_{2}$ is $200 \mathrm{~nm}$ ) substrate with markers patterned lithographically, which is commonly used in electron-beam lithography (EBL), was cleaned with acetone. Then, some nanoribbons

were individually removed from the original grown substrate and transferred onto the pre-cleaned $\mathrm{Si} / \mathrm{SiO}_{2}(200 \mathrm{~nm})$ substrate via homemade micromanipulation technique [47], following which a dielectric cladding of $\mathrm{HfO}_{2}$ (15 nm thick) was deposited on the dispersed nanoribbons through atomic layer deposition (ALD) method. Finally, Au nanodisk arrays (thickness $\sim 50 \mathrm{~nm}$ ) were fabricated on some nanoribbons by EBL 
(JOEL, JSM-6510) using a standard liftoff procedure [48]. A representative hybrid nanostructure is schematically described in Fig. 1(a).

\section{Morphology characterization and optical measurement}

Fig. 1(b) is the scanning electron microscopy (SEM) image for a representative hybrid nanostructure with three rows Au nanodisk array along the length of the ribbon, which shows that Au nanodisks, with nearly the uniform diameter of $\sim 300 \mathrm{~nm}$, were aligned on the $\mathrm{CdS}$ nanoribbon into three rows. Fig. 1(c) gives the Atomic Force Microscopy (AFM) image of the sample same as in Fig. 1(b). The further analysis of the AFM results with cross section profile (see Fig. 1(d)) along the marked line in Fig. 1(c) indicates that the thickness of the CdS nanoribbon and Au nanodisk is $\sim 100 \mathrm{~nm}$ and $\sim 48 \mathrm{~nm}$, respectively.

The photoluminescence (PL) spectra and optical waveguide were conducted on a Confocal Microscope (Witec $\alpha$-SNOM 300), locally excited with a $488 \mathrm{~nm}$ laser (Ar+ laser). Fig. 2(a) shows the representative PL spectrum of bare CdS nanoribbons. The single band emission with the peak wavelength at $510 \mathrm{~nm}$ is consistent with the band-edge emission of CdS at room temperature, which indicates the high quality of the CdS nanoribbon. Fig. 2(b) and (c) give the dark field optical micrographs of a bare CdS nanoribbon respectively under wide illumination of tungsten lamp and local excitation of $488 \mathrm{~nm}$ laser. Except for the light spot emitted from the excited point, the light can be guided to and emits from the end of the ribbon, which presents a monolithic rectangular light strip at the right end (see the amplified image of the white line marked area in Fig. 2(c)).This is due to the high refractive index contrast between 
the nanoribbon and surroundings, the nanoribbon can function as a rectangle optical waveguide cavity, in which light can be well confined and guided along its length direction. According to the waveguide theories, the evanescent wave should exist on the surface of the waveguide nanoribbon, and hence interacts with the Au nanodisks fabricated on the ribbon. Fig. 2(d) shows the dark field optical micrograph of a CdS nanoribbon with two rows of Au nanodisks array under the wide illumination of tungsten lamp, from which the individual $\mathrm{Au}$ nanodisks can be distinguished. Comparing with the bare CdS nanoribbon, under locally excitation with focused 488 $\mathrm{nm}$ laser, the ribbon with two rows of Au nanodisks shows three unambiguous light spots at its terminal end isolated with two dark regions just corresponding to the position of the Au nanodisk rows (see the right end in Fig. 2(e)). The number of the waveguide light spots at the end of $\mathrm{CdS}$ nanoribbon can be tuned by varying the number of the Au nanodisks array rows. Fig. 2(f) shows the dark field image of the $\mathrm{CdS}$ nanoribbon with three rows of $\mathrm{Au}$ nanodisks under wide illumination, and the corresponding optical image with focused laser excitation is given in Fig. 2(g). Four light spots can be clearly observed, and isolated by three dark region at the position corresponding the Au rows. Therefore, we conclude that the Au nanodisks rows on CdS nanoribbon can split the waveguide light beam, which is attributed to the interaction between Au nanodisk arrays and the guided light in the ribbon.

\section{Simulations and discussion}

To obtain further insight into the light-matter interaction mechanism in this novel nanostructure, numerical simulations were carried out using the finite element method 
$[6,49]$. Herein, basing on the size of the fabricated sample, we build a $3 \mathrm{D}$ model of the hybrid nanostructure with two rows of Au nanodisks array on the CdS nanoribbon, where a z-axis polarized light of $510 \mathrm{~nm}$ was launched from one end of the ribbon [50]. The theoretical model is based on the equation: $\boldsymbol{\nabla} \times(\boldsymbol{\nabla} \times \mathbf{E})-k_{0}^{2} \varepsilon_{\mathrm{r}} \mathbf{E}=0$ [51], where $\mathbf{E}$ is the electric fields of the optical field and the complex relative permittivity $\varepsilon_{\mathrm{r}}=\varepsilon_{\mathrm{r}}^{\prime}+\varepsilon_{\mathrm{r}}^{\prime \prime}=(n+i k)^{2}$ is determinded by the refractive index $n$ and the extinction coefficient $k$ of the material, with real and imaginary parts $\varepsilon_{\mathrm{r}}^{\prime}$ and $\varepsilon_{\mathrm{r}}^{\prime \prime} . \mathrm{k}_{0}=\omega / c$ is the free space wave number, $\omega$ is the angular frequency, $c$ is the speed of light in vacuum. The material of each part of the nanostructure was defined by the wavelength dependent $n$ and $k . n$ of $\mathrm{CdS}$ nanoribbon, $\mathrm{HfO}_{2}$ dielectric layer and Au nanodisikes are $2.82,2.11$ and 0.75 , respectively, and $k$ of $\mathrm{Au}$ was 1.92 . For illustrating the optical splitting mechanism of the hybrid nanostructure qualitatively, we provide the calculated results of the simplified simulation model without containing the substrate and set the surrounding medium of the nanostructure as air $(n=1)$. Fig. 3(a) is the volume electric field (E-field) distribution at the surface of the simulated structure, three maximum E-field regions along the length of the ribbon are observed, which are isolated by two dark regions just corresponding to each row of the Au nanodisks. This can be further proved by the cross section profile analysis for the down end of the ribbon (see inset of Fig. 3(a)), as well as the mode profiles of $x-z$ and $y-z$ plane are shown in Fig. 3(b). Especially, the mode profile of y-z plane in bottom of Fig. 3(b) shows that the maximum E-field distributes at the regions without Au nanodisks, and the minimum one is in the ribbon under the Au nanodisks, which clearly demonstrates 
surface attached $\mathrm{Au}$ nanodisks absorb the guided light beneath it in the nanoribbon. Fig. 3(c) shows the surface charge density distribution simulation image of one representative Au nanodisk, which indicates the charge at the surface of Au nanodisk is polarized. Surface plasmons are generated from the interaction between the light wave trapped at metal surface and free electrons in the metal $[52,53]$. Here, the $\mathrm{HfO}_{2}$ thin film was used as the spacer to avoid the detrimental effect of nonradiative surface trap states caused by the Au nanodisks on the CdS nanoribbon, which offers the opportunity to investigate the intrinsic properties of the CdS ribbons $[54,55]$. Additionally, because of the sub-wavelength size and the relative large interval of $\mathrm{Au}$ nanodisks, plasmons can not propagate along the metal-dielectric interface but oscillate locally around the Au nanodisks, which is so called localized plasmon (LSP) [56]. Therefore, the light guided in the nanoribbon is trapped by the Au nanodiscks, which results in the collective oscillation of free electrons. Due to the scattering loss and inherent ohmic damping of the metal, much of the absorbed energy is dissipated in these Au nanodisks. As a result, it is reasonable to believe that the propagating light through the areas covered with Au nanodisks will be absorbed by Au arrays, by which $\mathrm{Au}$ arrays isolate the light propagated in the nanoribbon.

To such a nanostructure, optical waveguide in the nanoribbon can be considered as several light beams propagating along each sub-wavelength channel (about $300 \mathrm{~nm}$ width) isolated by Au nanodisks array. The rule that the number of Au nanodisks array rows plus one equals the number of light beams is obviously manifest by the light-matter interaction mechanism in this nanostructure. Although the light beams are 
at sub-wavelength scale, there are both effect of optical diffraction and multi-beam interference similar to Young's interference taken place in the waveguide process [57]. As a result, the number of emitted light spots is not only determined by the number of Au nanodisks array rows, but also by the interference length of multi-beams (distance of the blank region between the nanodisks array and the terminal end of the CdS ribbon, (DC), illustrated in Fig. $4\left(a_{1}\right),\left(b_{1}\right)$ and $\left.\left(c_{1}\right)\right)$. Three representative samples that contain same rows of Au nanodisks array with different DC were measured. Fig. 4(a $\left.a_{1}\right)$ gives the SEM image of one sample which contains three rows of Au nanodisks with DC about $0.45 \mu \mathrm{m}$ and the corresponding dark field optical image shows that four light spots were emitted at the terminal end of CdS ribbon under locally excitation with focused $488 \mathrm{~nm}$ laser (Fig. 4(a $\left.\mathrm{a}_{2}\right)$ ). From its corresponding calculated cross-sectional (x-y plane) E-field distribution in the ribbon (Fig. 4(a $\left.\mathrm{a}_{3}\right)$ ), it is found that three rows of Au nanodisks have split the guided light into four optical beams, which is due to the DC is not long enough to induce apparent interference and four parted signal output at the terminal of the ribbon. However, for the sample with DC about $1.5 \mu \mathrm{m}$ (Fig. $4\left(\mathrm{~b}_{1}\right)$ ), five light spots were emitted at the terminal end of CdS ribbon under locally excitation with focused $488 \mathrm{~nm}$ laser (Fig. 4(b $\left.\mathrm{b}_{2}\right)$ ). This phenomenon indicates that the DC is long enough for interference between the four split beams, which is proved by its corresponding simulated result (Fig. 4(b $\left.b_{3}\right)$ ). Fig. 3(c) shows the sample with DC of about $2.2 \mu \mathrm{m}$, it is found six split light spots emitted at the terminal end of $\mathrm{CdS}$ ribbon (Fig. 3( $\left.\mathrm{c}_{2}\right)$ ), and the corresponding simulation results gives the interference pattern resulted from four split light beams 
(Fig. 3( $\left.c_{3}\right)$ ). Therefore, it can be concluded that emitted light spots at the terminal end of nanoribbon is determined by light-matter interaction and the interference of light, which can be used as light splitter in nano-scale optical devices.

From the experimental and simulation results, good splitting ration of the fabricated nanostructure can be inferred from the approximately uniform emit light spots at the end of the nanoribbon and the similar E-field intensity peak values of the three maximum E-field regions (inset of Fig. 3(a)). The equation $\boldsymbol{\nabla} \times(\boldsymbol{\nabla} \times \mathbf{E})-\mathrm{k}_{0}^{2}{ }_{\mathrm{r}} \mathbf{E}=$ 0 indicates that the E-field intensity distribution in the nanostructure can be modulate by altering the relative dielectric constant of the material: $\varepsilon_{\mathrm{r}}=\varepsilon_{\mathrm{r}}^{\prime}+i \varepsilon_{\mathrm{r}}^{\prime \prime}=(n+i k)^{2}$. Moreover, the imaginary part $\varepsilon_{\mathrm{r}}^{\prime \prime}$ concerns the dissipation of energy. There are two parameters, the wavelength dependent refractive index $\mathrm{n}$ and the extinction coefficient $k$, determine the value of $\varepsilon_{\mathrm{r}}^{\prime \prime}$, which equals to $2 n k$. The dissipation of energy in metal is a major factor that affects the propagation loss of the splitter. Therefore, choosing the metal which has relative lower value of $n k$ may reduce the propagation loss. Additionally, low refractive index substrates benefit the guided light confining in the device, due to the large refractive index contrast between the waveguide cavity and the substrate.

\section{Conclusion}

In conclusion, we have designed and fabricated a type of sandwich-like hybrid metal-dielectric-semiconductor optical waveguide beam splitters, which can split the waveguide light into several beams emitting at the end of the CdS nanoribbon. Corresponding numerical simulations demonstrate that waveguide in the nanoribbon 
is coupled with the free electrons of the upper Au nanodisks to generate LSP, due to the existence of metal array. This interaction leads to confine and guide light in several sub-wavelength scale channels isolated by $\mathrm{Au}$ nanodisks arrays in this nanostructure. The experiment and numerical simulation results reveal that the number of light spots can be tuned by adjusting the number of Au nanodisks array rows and the interference length of the split multi-beams. This kind of nanostructure is a practical high-density optics device and may provide a significant progress for micro/nanoscale optical sensing, logical operation, and communication or compact optical components for integrated nanophotonics.

\section{Acknowledgments}

The authors are grateful to the NSF of China (Nos.11374092, 11204073, 61474040, and 51302077), the National Basic Research Program of China (No. 2012CB932703), the Hunan province science and technology plan (No.2014FJ2001, 2014GK3015, and 2014TT1004), the Hunan Provincial Natural Science Foundation of China (No.2015JJ3049), (CX2014B127) Supported by Hunan Provincial Innovation Foundation For Postgraduate and the Aid program for Science and Technology Innovative Research Team in Higher Educational Institutions of Hunan Province.

\section{References}

[1] R. F. Oulton, V. J. Sorger, T. Zentgraf, R. M. Ma, C. Gladden, L. Dai, G. Bartal, X. Zhang, Plasmon lasers at deep subwavelength scale, Nature 461 (2009) 629-632. 
[2] R. Colombelli, K. Srinivasan, M. Troccoli, O. Painter, C. F. Gmachl, D. M. Tennant, A. M. Sergent, D. L. Sivco, A. Y. Cho, F. Capasso, Quantum Cascade Surface-Emitting Photonic Crystal Laser, Science 302 (5649) (2003) 1374-1377.

[3] M. t. Hill, Y. Oei, B. Smalbrugge, Y. Zhu, T. De. Vries, P. J. V. Veldhoven, F. W. M. V. Otten, T. J. Eijkemans, J. P. Turkiewicz, H. D. Waardt, E. J. Geluk, S. Kwon, Y. Lee, R. N. Tzel1, M. K. Smit, Lasing in metallic-coated nanocavities, Nat. Photonics 1 (2007) 589-594.

[4] P. Guo, X. Zhuang, J. Xu, Q. Zhang, W. Hu, X. Zhu, X. Wang, Q. Wan, P. He, H. Zhou, A. Pan, Low-threshold nanowire laser based on composition-symmetric semiconductor nanowires , Nano Lett. 13 (3) (2013) 1251-1256.

[5] H. P. Paudel, M. N. Leuenberger, Light-Controlled Plasmon Switching Using Hybrid MetalSemiconductor Nanostructures, Nano Lett. 12 (6) (2012) 2690-2696.

[6] J. Ye, C. Zhang, C. L. Zou, Y. Yan, J. Gu, Y. S. Zhao, J. Yao, Optical wavelength filters based on photonic confinement in semiconductor nanowire homojunctions, Adv. Mater. 26 (4) (2014) 620-624.

[7] J. Feng, C. Zhou, J. Zheng, H. Cao, P. Lv, Dual-function beam splitter of a subwavelength fused-silic a grating, Appl. Optics. 48 (14) (2009) 2697-2701.

[8] J. Xu, X. Zhuang, P. Guo, Q. Zhang, W. Huang, Q. Wan, W. Hu, X. Wang, X. Zhu, C. Fan, Z. Yang, L. Tong, X. Duan, A. Pan, Wavelength-converted/selective waveguiding based on composition-graded semiconductor nanowires, Nano Lett. 12 (9) (2012) 5003-5007.

[9] X. Xing, H. Zhu, Y. Wang, B. Li, Ultracompact Photonic Coupling Splitters 
Twisted by PTT Nanowires, Nano Lett. 8 (9) (2008) 2839-2843.

[10] G. M. Lerman, U. Levy, Pin cushion plasmonic device for polarization beam splitting, focusing, and beam position estimation, Nano Lett. 13 (3) (2013) 1100-1105.

[11] J. S. Liu, R. A. Pala, F. Afshinmanesh, W. Cai, M. L. Brongersma, A submicron plasmonic dichroic splitter, Nat. Commun. 2 (525) (2011) 1-5.

[12] X. Ao, L. Liu, L. Wosinski, S. He, Polarization beam splitter based on a two-dimensional photonic crystal of pillar type, Appl. Phys. Lett. 89 (17) (2006) 171115.

[13] S. Seo, S. Cho, N. M. Jokerst, Integrated thin film InGaAsP laser and $1 \times 4$ polymer multimode interference splitter on silicon, Opt. Lett. 32 (5) (2007) 548-550.

[14] L. Zhou, W. Liu, Broadband polarizing beam splitter with an embedded metal-wire nanograting, Opt. Lett. 30 (12) (2005) 1434-1436.

[15] H. Li, X. Dong, E. Li, Z. Liu, Y. Bai, Design Optimization and Comparative Analysis of Silicon-Nanowire-Based Couplers, IEEE Photonics 4 (5) (2012) 2017-2028.

[16] H. Ditlbacher, J. R. Krenn, G. Schider, A. Leitner, F. R. Aussenegg, Two-dimensional optics with surface plasmon polaritons. Appl. Phys. Lett. 81 (10) (2002) 1762-1764. 
[17] S. I. Bozhevolnyi, V. S. Volkov, E. Devaux, J. Laluet, T. W. Ebbesen, Channel plasmon subwavelength waveguide components including interferometers and ring resonators, Nature 440 (2006) 508-511.

[18] M. I. Stockman, Nanofocusing of Optical Energy in Tapered Plasmonic Waveguides, Phys. Rev. Lett. 93 (13) (2004) 137404.

[19] H. Duan, A. I. Fernandez-Domínguez, M. Bosman, S. A. Maier, J. K. W. Yang, Nanoplasmonics: Classical down to the Nanometer Scale, Nano Lett. 12 (3) (2012) 1683-1689.

[20] K. Kumar, H. Duan, R. S. Hegde, S. C. W. Koh, J. N. Wei, J. K. W. Yang, Printing colour at the optical diffraction limit, Nat. Nanotechnol. 7 (2012) 557-561.

[21] Y. Yan, C. Zhang, J. Y. Zheng, J. Yao, Y. S. Zhao, Optical modulation based on direct photon-plasmon coupling in organic/metal nanowire heterojunctions, Adv. Mater. 24 (42) (2012) 5681-5686.

[22] S. Y. Lee, K. Nakaya, T. Hayashi, M. Hara, Quantitative study of the gold-enhanced fluorescence of $\mathrm{CdSe} / \mathrm{ZnS}$ nanocrystals as a function of distance using an AFM probe, Phys. Chem. Chem. Phys. 11 (21) (2009) 4403-4409.

[23] M. Haridas, L. N. Tripathi, J. K. Basu, Photoluminescence enhancement and quenching in metal-semiconductor quantum dot hybrid arrays, Appl. Phys. Lett. 98 (6) (2011) 063305 .

[24] H. A. Atwater, A. Polman, Plasmonics for improved photovoltaic devices, Nat. Mater. 9 (3) (2010) 205-213.

[25] A. J. Haes, W. P. Hall, L. Chang, W. L. Klein, R. P. V. Duyne, A Localized 14 
Surface Plasmon Resonance Biosensor: First Steps toward an Assay for Alzheimer's Disease, Nano Lett. 4 (6) (2004) 1029-1034.

[26] H. Wei, Z. Li, X. Tian, Z. Wang, F. Cong, N. Liu, S. Zhang, P. Nordlander, N. J. Halas, H. Xu, Quantum dot-based local field imaging reveals plasmon-based interferometric logic in silver nanowire networks, Nano Lett. 11 (2) (2011) 471-475.

[27] N. Liu, H. Wei, J. Li, Z. Wang, X. Tian, A. Pan, H. Xu, Plasmonic amplification with ultra-high optical gain at room temperature, Sci. Rep. 3 (1967) (2013) 1-7.

[28] F. Abbas, Q. A. Naqvi, M. Faryad, Multiple surface plasmon-polariton waves guided by the interfaceof a metal and a periodically nonhomogeneous magnetic material, Opt. Commun. 332 (2014) 109-113.

[29] S. K. R. Namasivayam, G. K. E, R. R, Synthesis of silver nanoparticles by Lactobaciluus acidophilus 01 strain and evaluation of its in vitro genomic DNA toxicity, Nano-Micro Lett. 2 (3) (2010) 160-163.

[30] William L. Barnes, Alain Dereux, Thomas W. Ebbesen, Surface plasmon subwavelength optics, Nature 424 (2003) 824-830.

[31] A. Pan, R. Liu, Q. Zhang, Q. Wan, P. He, M. Zacharias, B. Zou, Fabrication and Red-Color Lasing of Individual Highly Uniform Single-Crystal CdSe Nanobelts, J. Phys. Chem. C. 111 (38) (2007) 14253-14256.

[32] Z. W. Pan, Z. R. Dai, Z. L. Wang, Nanobelts of Semiconducting Oxides, Science 291 (5510) (2001) 1947-1949.

[33] F. Gu, Z. Yang, H. Yu, J. Xu, P. Wang, L. Tong, A. Pan, Spatial bandgap 
engineering along single alloy nanowires, J. Am. Chem. Soc. 133 (7) (2011) 2037-2039.

[34] J. Qi, W. Liu, C. Biswas, G. Zhang, L. Sun, Z. Wang, X. Hu, Y. Zhang, Enhanced power conversion efficiency of CdS quantum dot sensitized solar cells with $\mathrm{ZnO}$ nanowire arrays as the photoanodes, Opt. Commun. 349 (2015) 198-202.

[35] L. Jia, M. Geng, L. Zhang, L. Yang, P. Chen, T. Wang, Y. Liu, Wavelength conversion based on degenerate-four-wave-mixing with continuous-wave pumping in silicon nanowire waveguide, Opt. Commun. 282 (8) (2009) 1659-1663.

[36] X. Y. Han, Y. H. Gao, X. H. Zhang, One-dimensional GaN nanomaterials transformed from one-dimensional $\mathrm{Ga}_{2} \mathrm{O}_{3}$ and $\mathrm{Ga}$ nanomaterials, Nano-Micro Lett. 1 (1) (2009) 4-8.

[37] M. H. Huang, S. Mao, H. Feick, H. Yan, Y. Wu, H. Kind, E. Weber, R. Russo, P. Yang, Room-temperature ultraviolet nanowire nanolasers, Science 292 (5523) (2001) 1897-1899.

[38] R. Liu, Z. A. Li, C. Zhang, X. Wang, M. A. Kamran, M. Farle, B. Zou, Single-step synthesis of monolithic comb-like $\mathrm{CdS}$ nanostructures with tunable waveguide properties, Nano Lett. 13 (6) (2013) 2997-3001.

[39] S. Nie, S. R. Emory, Probing Single Molecules and Single Nanoparticles by Surface-Enhanced Raman Scattering, Science 275 (5303) (1997) 1102-1106.

[40] H. Yu, W. Fang, X. Wu, X. Lin, L. Tong, W. Liu, A. Wang, Y. R. Shen, Single nanowire optical correlator, Nano Lett. 14 (6) (2014) 3487-3490.

[41] By Liang Li, Peicai Wu, Xiaosheng Fang, Tianyou Zhai, Lun Dai, Meiyong 
Liao, Yasuo Koide, Hongqiang Wang, Yoshio Bando, Dmitri Golberg, Single-Crystalline CdS Nanobelts for Excellent Field-Emitters and Ultrahigh Quantum-Efficiency Photodetectors, Adv. Mater 22 (2010) 3161 - 3165.

[42] A. Apuzzo, M. Fevrier, R. Salas-Montiel, A. Bruyant, A. Chelnokov, G. Lerondel, B. Dagens, S. Blaize, Observation of near-field dipolar interactions involved in a metal nanoparticle chain waveguide, Nano Lett. 13 (3) (2013) 1000-1006.

[43] Z. Fang, C. Lin, R. Ma, S. Huang, X. Zhu, Planar Plasmonic Focusing and Optical Transport Using CdS Nanoribbon, ACS. nano 4(1) (2010) 75-82.

[44] Q. Zhang, X. Y. Shan, X. Feng, C. X. Wang, Q. Q. Wang, J. F. Jia, Q. K. Xue, Modulating resonance modes and Q value of a CdS nanowire cavity by single Ag nanoparticles, Nano Lett. 11 (10) (2011) 4270-4274.

[45] A. Casadei, E. F. Pecora, J. Trevino, C. Forestiere, D. Ruffer, E. Russo-Averchi, F. Matteini, G. Tutuncuoglu, M. Heiss, A. Fontcuberta. I. Morral, L. Dal. Negro, Photonic-plasmonic coupling of GaAs single nanowires to optical nanoantennas, Nano Lett. 14 (5) (2014) 2271-2278.

[46] A. Pan, D. Liu, R. Liu, F. Wang, X. Zhu, B. Zou, Optical waveguide through CdS nanoribbons, Small 1 (10) (2005) 980-983.

[47] Pengfei Guo, Wei Hu, Qinglin Zhang, Xiujuan Zhuang, Xiaoli Zhu, Hong Zhou, Zhengping Shan, Jinyou Xu, Anlian Pan, Semiconductor Alloy Nanoribbon Lateral Heterostructures for High-Performance Photodetectors, Adv. Mater 26 (2014) 2844-2849. 
[48] X. Zou, J. Wang, C. Chiu, Y. Wu, X. Xiao, C. Jiang, W. Wu, L. Mai, T. Chen, J. Li, J. C. Ho, L. Liao, Interface Engineering for High-Performance Top-Gated $\mathrm{MoS}_{2}$ Field-Effect Transistors, Adv. Mater. 26 (2014) 6255-6261.

[49] F. Gu, L. Zhang, G. Wu, Y. Zhu, H. Zeng, Sub-bandgap transverse frequency conversion in semiconductor nano-waveguides, Nanoscale 6 (21) (2014) $12371-12375$.

[50] L. Sun, H. Kim do, K. H. Oh, R. Agarwal, Strain-induced large exciton energy shifts in buckled CdS nanowires, Nano Lett. 13 (8) (2013) 3836-3842.

[51] K. Kawano, T. Kitoh, Introduction to Optical Waveguide Analysis, John Wiley \& Sons, New York, 2001.

[52] E. Laux, C. Genet, T. Skauli, T. W. Ebbesen, Plasmonic photon sorters for spectral and polarimetric imaging, Nat. Photonics 2 (3) (2008) 161-164.

[53] J. Yoo, X. Ma, W. Tang, G. C. Yi, Metal-lined semiconductor nanotubes for surface plasmon-mediated luminescence enhancement, Nano Lett. 13 (5) (2013) 2134-2140.

[54] L. Tang, J. Xu, P. Guo, X. Zhuang, Y. Tian, Y. Wang, H. Duan, A. Pan, Modulated exciton-plasmon interactions in $\mathrm{Au}-\mathrm{SiO}_{2}-\mathrm{CdTe}$ composite nanoparticles, Opt. Express 21 (9) (2013) 11095-11100.

[55] P. Guo, J. Xu, X. Zhuang, W. Hu, X. Zhu, H. Zhou, L. Tang, A. Pan, Surface plasmon resonance enhanced band-edge emission of $\mathrm{CdS}-\mathrm{SiO}_{2}$ core-shell nanowires with gold nanoparticles attached, J. Mater. Chem. C. 1 (3) (2013) $566-571$. 
[56] K. A. Willets, R. P. Van Duyne, Localized surface plasmon resonance spectroscopy and sensing, Annu. Rev. Phys. Chem. 58 (2007) 267-297.

[57] L. Sun, M. L. Ren, W. Liu, R. Agarwal, Resolving parity and order of Fabry-Perot modes in semiconductor nanostructure waveguides and lasers: Young's interference experiment revisited, Nano Lett. 14 (11) (2014) 6564-6571.

Figures
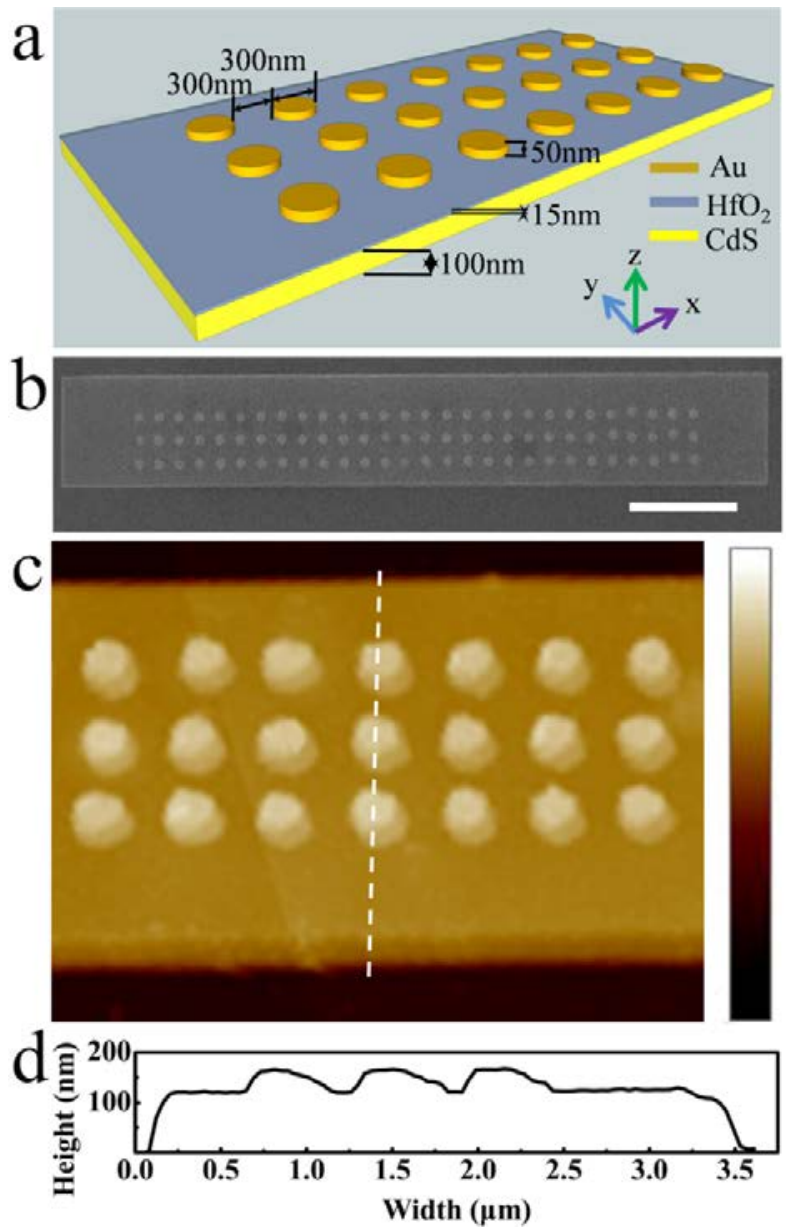

Fig. 1. (a) Schematic of the semiconductor-dielectric-metal beam splitter. A nanodisk array consist of Au cylindrical nanodisks with $300 \mathrm{~nm}$ diameter and $50 \mathrm{~nm}$ height, a 
100-nm-thick CdS nanoribbon and a $15 \mathrm{~nm}$ thick $\mathrm{HfO}_{2}$ layer are plotted from top to bottom. Period of the Au nanodisk array is $600 \mathrm{~nm}$. (b) SEM image of a representative sample (scale bar is $3 \mu \mathrm{m}$ ). (c) AFM morphological image of the sample (color scale bar indicates the height of the sample from 0 to 170). (d) Cross-section profile plot of the sample along the white dashed line marked in (c). 

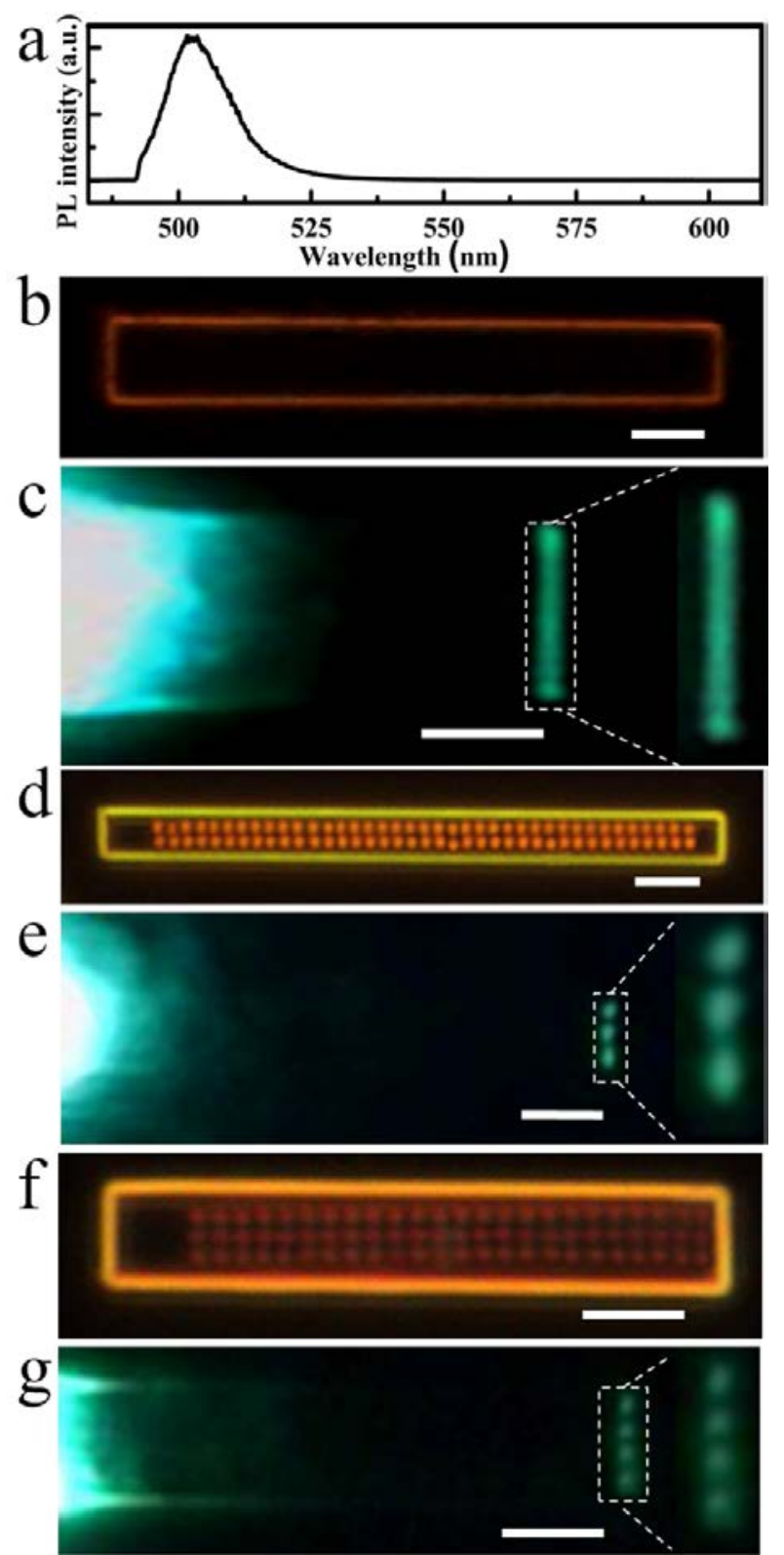

Fig. 2. (a) The representative PL spectrum of CdS nanoribbons. (b) Dark field optical micrograph of a $\mathrm{CdS}$ nanoribbon without $\mathrm{Au}$ nanodisks under wide illumination of tungsten lamp. (c) Dark field optical micrograph of the bare ribbon under local excitation of $488 \mathrm{~nm}$ laser. (Both of the scale bars are $4 \mu \mathrm{m}$ ). (d) Dark field optical micrograph of a representative sample contains two rows of Au nanodisks array under wide illumination of tungsten lamp (scale bar is $2 \mu \mathrm{m}$ ). (e) Dark field optical 
micrograph of the sample under local excitation of $488 \mathrm{~nm}$ laser. Inset is the amplified image of the terminal end of CdS nanoribbon (scale bar is $2 \mu \mathrm{m}$ ). (f) Dark field optical micrograph of a sample containing three rows of Au nanodisks array under wide illumination of tungsten lamp (scale bar is $3 \mu \mathrm{m}$ ). (g) Dark field optical micrograph of the sample under local excitation of $488 \mathrm{~nm}$ laser. Inset is the amplified image of the terminal end of CdS nanoribbon (scale bar is $3 \mu \mathrm{m}$ ).
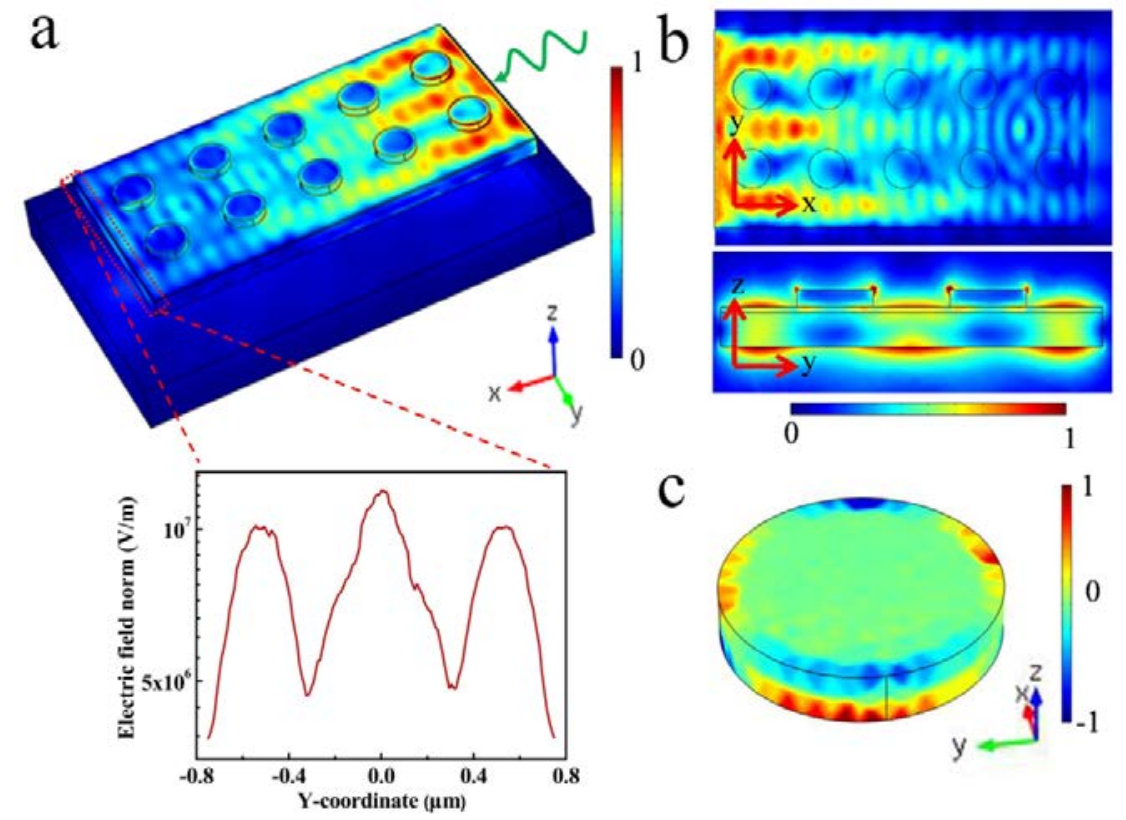

Fig. 3. (a) 3D-simulation of E-field distributions for the sample with guided modes at wavelength of $510 \mathrm{~nm}$. Inset is the E-field distributions profiles along the width direction at the terminal end of the CdS nanoribbon marked with dashed red line. (b) the simulated cross-sectional ( $\mathrm{x}-\mathrm{y}$ plane and $\mathrm{y}-\mathrm{z}$ plane) E-field distribution in $\mathrm{CdS}$ nanoribbon (y-z plane was plot just along the center at one rank of Au nanodisks). (c) The charge distribution of one representative Au nanodisk. 


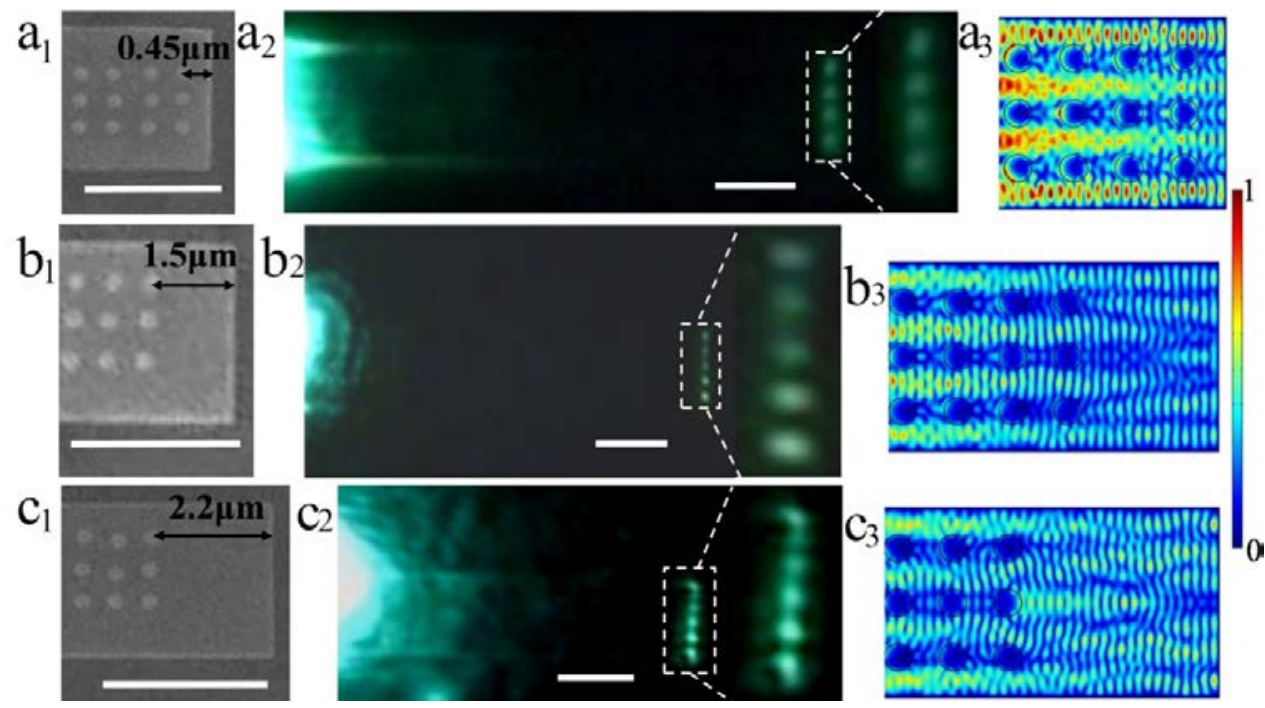

Fig. 4. Correlation between the number of emitted light spots and DC. $\left(a_{1}\right),\left(b_{1}\right)$ and $\left(c_{1}\right)$, the SEM images of the terminal end of the sample containing three rows of Au nanodisk array with DC of about $0.45 \mu \mathrm{m}, 1.5 \mu \mathrm{m}$ and $2.2 \mu \mathrm{m}$, respectively. $\left(\mathrm{a}_{2}\right),\left(\mathrm{b}_{2}\right)$ and $\left(c_{2}\right)$ are their dark field optical micrographs under the local excitation of $488 \mathrm{~nm}$ laser, (all of the scale bars are $3 \mu \mathrm{m}) .\left(a_{3}\right),\left(b_{3}\right)$ and $\left(c_{3}\right)$, the calculated E-field distribution for the cross-section ( $\mathrm{x}-\mathrm{y}$ plane) of corresponding hybrid structures. 